\title{
ESTUDO DE NECESSIDADES PARA O DESIGN DE INTERFACE DE WEB APLICATIVO PARA ASSISTÊNCIA A ANIMAIS NECESSITADOS
}

\section{REQUIREMENTS STUDY FOR THE INTERFACE DESIGN OF WEB APPLICATION FOR ASSISTANCE TO ANIMALS IN NEED}

\author{
Jessica Aparecida Freitas Peixoto ${ }^{1}$ \\ Marina Giolo Passos ${ }^{2}$ \\ Gabriela Costa Mendes ${ }^{3}$ \\ Ravi Passos ${ }^{4}$, Dr.
}

(1) Universidade Federal de Goiás, e-mail: jessica.fpeixoto@gmail.com

(2) Universidade Federal de Goiás, e-mail: $\underline{\text { marih.design@gmail.com }}$

(3) Universidade Federal de Goiás, e-mail: gabcmendes@gmail.com

(4) Universidade Federal de Goiás, e-mail: ravipassos@ ravipassos.com

Palavras-chave: design de interfaces, interação, resgate de animais.

\begin{abstract}
O presente estudo enquadra-se no contexto do levantamento de requisitos para design de interfaces para aplicativo de dispositivo móvel. Em particular, busca-se a contribuição desta área para a concepção e desenvolvimento de um aplicativo de assistência ao resgate e adoção de animais que possibilite a troca de informações mais rápida e eficaz. A partir de uma abordagem voltadas às necessidades dos usuários, utiliza-se da revisão bibliográfica acerca dos conceitos relacionados ao tema proposto, assim como análise de similares e entrevista com usuários. Como resultado, determinam-se sistematicamente os requisitos para o desenvolvimento de uma interface interativa, relacionando a fundamentação teórica ao conhecimento e desenvolvimento do artefato, em vista de uma solução que favoreça um problema social de abandono e maus tratos de animais domésticos.
\end{abstract}

Key-words: interface design, interaction, animal rescue.

The present study fits in the context of the survey of requirements for interface design for mobile application. In particular, we seek the contribution of this area to the design and development of an animal rescue and adoption assistance application that enables faster and more efficient exchange of information. Based on a user-oriented approach, the bibliographic review of the concepts related to the proposed theme is used, as well as the analysis of similar ones and interviews with users. As a result, the requirements for the development of an interactive interface are systematically determined, relating the theoretical basis to the knowledge and development of the object, seeking a solution that favors a social problem of abandonment and mistreatment of domestic animals 


\section{$16^{\circ}$ \\ ERGODESIGN USIHC CINAHPA}

\section{Introdução}

A incidência de animais abandonados e em situação de risco é recorrente e preocupante, nas mais diversas cidades do país, visto que se trata, também, de um problema de saúde pública. Isso porque, além das zoonoses que afetam a vida da população direta e indiretamente, é crime o abandono e maus tratos de animais (LEI ${ }^{\circ}$ 24.645). Existem, ainda, problemas de comunicação nas plataformas de divulgação de casos de resgate e adoção atuais, que se dão principalmente por meio das redes sociais, como demora, baixa visibilidade das publicações e falta de incentivo à conscientização em relação à saúde e bem-estar animal. Nesse sentido, os aplicativos para dispositivos móveis têm se revelado como potenciais ferramentas estratégicas para o estímulo de hábitos responsáveis, pois além das possibilidades de interação que eles oferecem aos usuários e a facilidade de acesso a esses dispositivos móveis, há também a possibilidade de educar, informar e conscientizar as pessoas sobre o tema em questão.

Por meio do design, é possível colaborar na solução de tal problema por meio do planejamento de interfaces para dispositivos móveis que auxiliem, desde o resgate dos animais em questão até a etapa considerada final, que seria a adoção. $\mathrm{O}$ tratamento de conteúdo permite, no intuito de otimizar a experiência no uso de interfaces, maximizar os conhecimentos a respeito do problema e envolver os usuários nas questões que o permeiam, transformando o usuário na peça essencial e principal da causa.

Neste trabalho, portanto, o design se insere de modo contributivo, com o objetivo de solucionar problemas de comunicação e interação, reunindo questões relacionadas à temática e levando em consideração as necessidades e características dos usuários tanto na organização e configuração do conteúdo quanto no desenvolvimento de uma interface de aplicativo prático e eficaz. $16^{\circ}$ Ergodesign - Congresso Internacional de Ergonomia e Usabilidade de Interfaces Humano Tecnológica: Produto, Informações Ambientes Construídos e Transporte

$16^{\circ}$ USIHC - Congresso Internacional de Ergonomia e Usabilidade de Interfaces Humano Computador

CINAHPA | 2017 - Congresso Internacional de Ambientes Hipermídia para Aprendizagem.

\section{Fundamentação teórica}

Conceitos e fundamentos teóricos serão discutidos a seguir, constituindo o alicerce para a definição de requisitos que orientam o desenvolvimento de interface, essencial ao escopo deste trabalho.

\subsection{Elementos de design}

Em interfaces gráficas, as tipografias podem assumir diferentes funções, tais como organizar informações, criar hierarquia, estabelecer uma ordem de leitura e expressar conceitos. Isso se dá por meio da utilização de diferentes famílias tipográficas, estilos, pesos e tamanhos, sem exagero para não criar impressão de ruído (GALITZ, 2002). Os textos para interfaces devem ser escritos de modo claro e simplificado, já que a leitura de itens na tela do computador ou de um dispositivo móvel é mais cansativa do que no papel (DAMASCENO, 2003).

Percepções, sensações e sentimentos gerados por cores devem ser levados em consideração no seu uso de acordo com a intenção que se deseja transmitir, portanto o uso de cores não deve ser feito de forma aleatória. O uso de cores atua como mecanismo de separação de grupos e categorias, e ainda, existem algumas questões que devem ser consideradas quando do seu uso em interfaces, tais como: sua aplicação deve ter um propósito e ser consistente, considerando o contexto de projeto para a escolha; não deve ser feito o uso de um número distinto excessivo, é recomendado o uso de modo padronizado e; é importante manter contraste entre os elementos e o fundo. (GALITZ, 2002)

Samara (2013, p.22), afirma que "um grid introduz uma ordem sistemática num leiaute, diferenciando tipos de informação e facilitando a navegação entre eles". No contexto do design de interfaces utilizase "o termo wireframe com papel equivalente ao do grid: indicar o local de conteúdo em uma interface, colaborando em suas relações" (PASSOS, 2014, p.70). Assim como o grid, o wireframe é um "esqueleto" do projeto de design, cuja construção também é baseada em linhas, colunas e formas geométricas e, além disso, é elaborado para 


\section{$16^{\circ}$ \\ ERGODESIGN USIHC CINAHPA}

organizar os elementos visuais e textuais do artefato final (idem).

\subsection{Interfaces digitais interativas}

Interfaces têm por objetivo a criação de um ponto de interconexão entre o usuário e a tecnologia, para um sistema de interação entre o homem e a máquina (LÉVY, 1998). Shneiderman (2010) propõe oito princípios para interfaces eficientes, intitulados pelo autor como "Oito Regras de Ouro de Design de Interface". São eles: esforço pela consistência, ou seja, padronização de elementos de design em situações semelhantes; reconhecer as necessidades de diferentes usuários em seus diferentes níveis de experiência; oferecer feedback informativo; planejar diálogos para encerrar ações; prevenir erros; permitir fácil reversão de ações; permitir que os usuários se sintam no controle do comando de ações; e evitar que o usuário precise memorizar muitas informações.

A interação pode ser considerada mútua ou reativa que, em termos de interface, significa que sistemas interativos mútuos se 'interfaceiam' virtualmente, em que há um complexo de problemas a serem solucionados; enquanto os sistemas reativos apresentam uma interface potencial, que é um conjunto de possibilidades que aguardam por sua realização. Em outras palavras, a interação mútua é a comunhão, estabelecida através de diálogo, ela se dá através de ações independentes; enquanto a reativa é um sistema interativo que deveria dar total autonomia ao espectador, porém o reagente tem pouca ou nenhuma condição de alterar o agente por apresentar relações lineares e unilaterais, esta é oposta à interação humana. (PRIMO, 2000)

\subsection{Ergonomia e usabilidade}

A ergonomia é definida pela IEA (2000, n.p) como a ciência que estuda as "interações entre os seres humanos e outros elementos ou sistemas e à aplicação de teorias, princípios, dados e métodos a projetos a fim de otimizar o bem-estar humano e o desempenho global do sistema" (ABERGO, s.d). Já a usabilidade, como explica Winckler \& Pimenta (2002) é uma área que estuda quão fácil é $16^{\circ}$ Ergodesign - Congresso Internacional de Ergonomia e Usabilidade de Interfaces Humano Tecnológica: Produto, Informações Ambientes Construídos e Transporte

$16^{\circ}$ USIHC - Congresso Internacional de Ergonomia e Usabilidade de Interfaces Humano Computador

CINAHPA | 2017 - Congresso Internacional de Ambientes Hipermídia para Aprendizagem. o manejo, acesso e compreensão de uma interface para seu usuário, em suma, é o que estuda a qualidade de uso desta.

Nielsen (2012) apresenta cinco qualidades que definem a usabilidade, são elas: facilidade de compreensão; eficiência, ou seja, quão fácil é para o usuário completar tarefas em geral; memorização, que diz respeito à quão fácil o usuário consegue reconhecer e encontrar o que deseja; erros, ou seja, quão facilmente os erros cometidos poder ser convertidos; e satisfação, em relação a interação com do usuário com a interface.

\section{Estudo do objeto}

Nesta etapa foram coletados e analisados dados correspondentes ao contexto em que se insere o objeto, no caso do presente trabalho uma interface para o resgate e adoção de animais, com finalidade de confirmar aspectos determinados anteriormente e estabelecer novos elementos que estruturaram a definição dos requisitos para o desenvolvimento do artefato. A metodologia utilizada, que é uma abordagem cartesiana dividida em etapas (PASSOS, 2014), propõe para isso o estudo de similares, o estudo de materiais e tecnologias e o estudo dos usuários como subetapas essenciais ao estudo do objeto.

\subsection{Estudo de similares}

O estudo de similares compreende uma análise comparativa entre os objetos similares ao objeto estudado. Para isso, foi utilizada a análise paramétrica, que é uma ferramenta eficaz para o propósito de determinar padrões a serem aplicados no desenvolvimento do artefato.

Os aplicativos escolhidos para esta análise foram Waze, Facebook, Tinder, Instagram, Twitter e Adote Pets, sendo a escolha devida à popularidade e qualidade dos mesmos e levando também em consideração aspectos relevantes e que são passíveis de aplicação ao produto final deste trabalho, tais como colaboratividade, compartilhamento de informações, uso de geolocalização, semelhança de temática (no caso do Adote Pets) e interação entre usuários.
Realização:

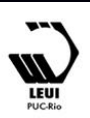




\section{$16^{\circ}$ \\ ERGODESIGN USIHC CINAHPA}

A sistematização da análise paramétrica se dá através da definição de parâmetros referentes ao tema de estudo, sendo que cada parâmetro possui suas variáveis e estas, por sua vez, sua definição. Cada um dos similares foi analisado e, em seguida, comparou-se os resultados obtidos (tabela 1).

\begin{tabular}{|c|c|c|}
\hline Parâmetro & Variáveis & Descrição \\
\hline \multirow[t]{3}{*}{ Usabilidade } & Tipo de interação & Interação reativa \\
\hline & Navegabilidade & Vertical, rolagem \\
\hline & Retomada & Sim, através de um botão \\
\hline \multirow{2}{*}{$\begin{array}{l}\text { Estrutura da } \\
\text { Informação }\end{array}$} & Tipo de grid & Modular \\
\hline & $\begin{array}{l}\text { Elementos que } \\
\text { favorecem a } \\
\text { hierarquia }\end{array}$ & Possui \\
\hline \multirow[t]{5}{*}{ Layout } & Cor & $\begin{array}{l}\text { Predominância cores } \\
\text { frias; contraste de cor } \\
\text { entre elementos e fundo é } \\
\text { alto; quantidade de cores } \\
\text { adequada; padronização } \\
\text { das cores é organizada }\end{array}$ \\
\hline & Tipografia & $\begin{array}{l}\text { Sem serifa, modulação } \\
\text { nula, pesos light e bold }\end{array}$ \\
\hline & $\begin{array}{l}\text { Ilustrações e } \\
\text { Grafismos }\end{array}$ & Geométricos \\
\hline & Estilo & Flat Design \\
\hline & $\begin{array}{l}\text { Botões, menus e } \\
\text { ícones }\end{array}$ & $\begin{array}{l}\text { Flat, menus expansíveis } \\
\text { localizados na barra } \\
\text { superior }\end{array}$ \\
\hline Tecnologia & Plataforma & $\begin{array}{l}\text { Android, iOS, Windows } \\
\text { Phone }\end{array}$ \\
\hline
\end{tabular}

Tabela 1: Conclusão da análise paramétrica.

\subsection{Estudo de materiais e tecnologias}

Existem diferentes abordagens de desenvolvimento atualmente: aplicativos nativos, web aplicativos, e aplicativos híbridos. Os nativos são desenvolvidos especificamente para uma determinada plataforma e utilizam as linguagens nativas de cada uma delas; já os web aplicativos são na realidade sites responsivos que utilizam linguagem de programação típica de web. Enquanto os nativos são instalados e armazenados dentro do dispositivo móvel e podem ser baixados através de lojas de $16^{\circ}$ Ergodesign - Congresso Internacional de Ergonomia e Usabilidade de Interfaces Humano Tecnológica: Produto, Informações Ambientes Construídos e Transporte

$16^{\circ}$ USIHC - Congresso Internacional de Ergonomia e Usabilidade de Interfaces Humano Computador

CINAHPA | 2017 - Congresso Internacional de Ambientes Hipermídia para Aprendizagem. aplicativos, os web aplicativos podem ser instalados na tela principal do dispositivo móvel criando um atalho para aquele site ou acessado via navegador, mas sua base é on-line. Os aplicativos híbridos são uma mescla dos dois outros. Eles utilizam linguagens de programação da web, podem ser baixados em lojas de aplicativos e utilizam recursos disponíveis no dispositivo. (SIMÃO et al., 2013). Assim sendo, web aplicativos são mais adequados no contexto de desenvolvimento deste trabalho, na medida em que podem ser disponibilizados em diferentes plataformas através de uma mesma linguagem de programação, tornando-se mais econômico e alcançando um maior número de usuários, além de serem mais 'leves' para armazenamento nos dispositivos.

\subsection{Estudo dos usuários}

Nesta etapa foi realizada uma pesquisa quantitativa para mensurar dados e identificar padrões quantificáveis sobre as características do públicoalvo, seus hábitos, gostos e opiniões relativos ao tema determinado e entrevistas qualitativas para esclarecer questões ainda em aberto, confirmar hipóteses e aprofundar o entendimento sobre aspectos e opiniões sobre os usuários. O principal objetivo é compreender as características e necessidades dos usuários e requisitos para o projeto do artefato em questão.

A pesquisa quantitativa foi realizada por meio da divulgação de um questionário estruturado na rede social Facebook em perfis pessoais, página de ONGs que acompanham casos de resgate e adoção de animais e grupos de assuntos diversos.

Obteve-se através do questionário quantitativo (Figura 1), que teve uma amostra de 937 indivíduos, sendo a maior parte do público interessado no assunto, que faz ou já fez resgates, e gosta muito de animais de estimação, sendo em sua maioria, mulheres entre 20 e 30 anos de classe social C (de acordo com a classificação estabelecida pelo IBGE). Também se esclareceu que a maior parte do público é tutor de um animal adotado ou ganhado, seguido por encontrado na rua, e que a maioria deles não são castrados. 


\section{$16^{\circ}$ \\ ERGODESIGN USIHC CINAHPA}

Apesar da quantidade superior de tutores com animais não castrados, a maioria tem acesso a grupos de resgate e campanhas de castração a baixo custo, principalmente através de páginas do Facebook. Foi analisado ainda, o interesse e acesso do público a smartphones, e concluiu-se haver predominância de pessoas que têm acesso, não sendo iniciantes e tendo interesse em aplicativos, inclusive sobre resgate e adoção de animais.

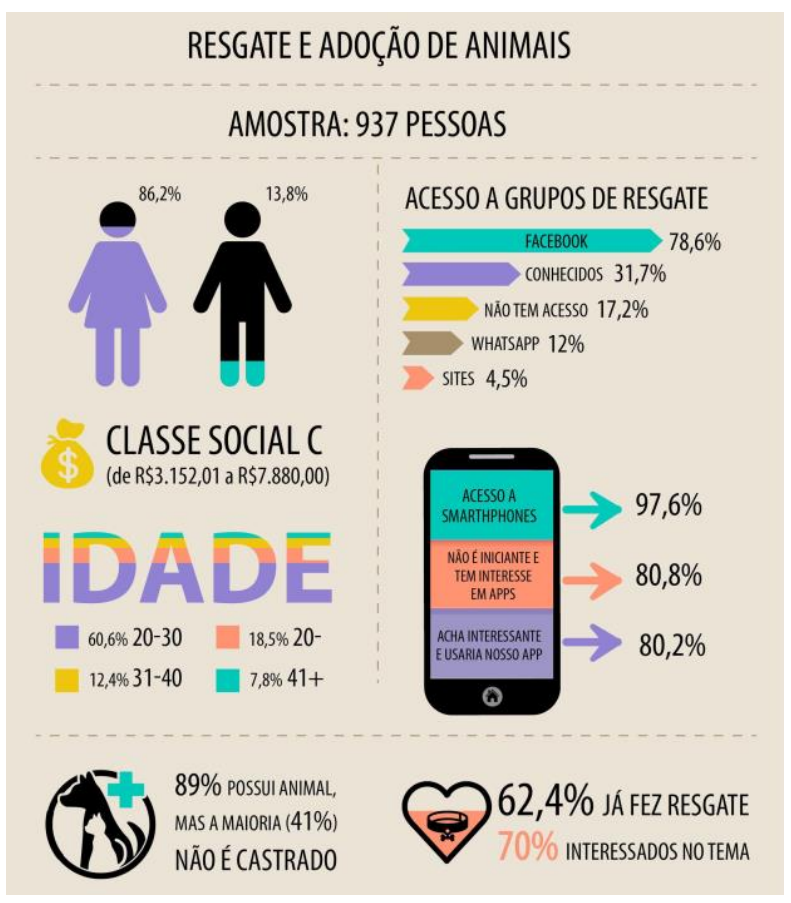

Figura 1: Infográfico dos resultados do questionário quantitativo.

Foram realizadas ainda, duas entrevistas por meio de perguntas estruturadas, com temas-chave envolvendo resgate, adoção, abrigo e abandono de animais, com dois especialistas sobre resgate e adoção de animais. Pode-se concluir com essas entrevistas, que existem problemas na divulgação dos casos pois os interessados não têm acesso a um formulário ou guia que lhes explique o que é necessário enviar como informação, além da demora e baixa visibilidade das divulgações dos casos. A problemática da disponibilização dos lares temporários deve ser levada em consideração, com o intuito de esclarecer que os animais em lar temporário não serão esquecidos lá, incentivando mais essa prática. Os entrevistados também relataram a importância da realização de triagem para adoção e acompanhamento destas, pois $16^{\circ}$ Ergodesign - Congresso Internacional de Ergonomia e Usabilidade de Interfaces Humano Tecnológica: Produto, Informações Ambientes Construídos e Transporte

$16^{\circ}$ USIHC - Congresso Internacional de Ergonomia e Usabilidade de Interfaces Humano Computador

CINAHPA | 2017 - Congresso Internacional de Ambientes Hipermídia para Aprendizagem.

diversas pessoas desistem do animal após adotá-lo e podem abandoná-los na rua. Foi citado, por fim, a importância de incentivar a castração, vacinação e vermifugação dos animais, assim como o uso de identificação através de plaquinhas e maior atenção com fugas.

\section{Definição de requisitos}

A partir da fundamentação teórica e estudo do objeto realizados, foram delimitados requisitos que têm a finalidade de orientar as decisões a serem tomadas durante o desenvolvimento do artefato de design, que no caso deste trabalho é uma interface para um web aplicativo colaborativo de assistência a animais necessitados. Assim sendo, esses requisitos foram organizados e agrupados de acordo com sua classificação como requisitos funcionais, formais e conceituais (PASSOS, 2014), assim dispostos:

Requisitos Funcionais: usar estilos distintos pesos e tamanhos de fonte para criar hierarquia e distinção visual; usar tipografia com boa legibilidade e leiturabilidade; criar hierarquia visual; ser responsivo; ter interação reativa e mútua; ser colaborativo; possuir geolocalização; ser multiplataforma; usar elementos gráficos (fotos, tabelas, grafismos, etc.); possuir elementos textuais simples, objetivos e de fácil compreensão; possuir botão de retorno; possuir link externo para redes sociais; possuir tutorial para iniciantes; contrastar tipografia e fundo; usar escala de cores hexadecimal/RGB; usar a linguagem do usuário de forma clara, simples e objetiva; oferecer feedback imediato; possuir gestão de erros; disponibilizar guias e formulários para preenchimento de pedidos de resgate ou adoção; realizar triagem e acompanhamento das adoções;

Requisitos Formais: predominância de estética feminina através de cores e formas; possuir tela de abertura (splash screen); usar ícones (pictogramas) para o menu principal; utilizar paleta de cores para enfatizar identidade visual; evitar excesso de cores; manter consistência e homogeneidade quanto aos elementos da interface;

Requisitos Conceituais: usar tipografias que
Realização:
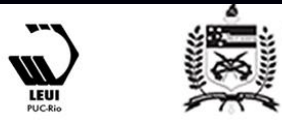


\section{$16^{\circ}$ \\ ERGODESIGN USIHC CINAHPA}

evidenciem confiança e credibilidade; favorecer colaboratividade; promover a importância de conscientização (resgate, lar temporário e adoção responsável); favorecer o acompanhamento dos casos.

\section{Considerações finais}

Esta pesquisa objetivou estudar e analisar o design de interfaces como orientador do levantamento de requisitos para o desenvolvimento de uma interface de aplicativo para tornar a troca de informações sobre resgate e adoção de animais mais rápida e eficaz. Para tal, utilizou-se a revisão bibliográfica acerca dos conceitos relacionados ao tema em questão, ao design de interface e um estudo do objeto, que no caso deste trabalho trata-se de um web aplicativo para resgate e adoção de animais.

Foi possível identificar a partir das informações coletadas no estudo do objeto que o tema resgate e adoção de animais ainda é pouco explorado na área do design de interfaces, sendo que mesmo os aplicativos existentes não envolvem a questão do resgate, etapa fundamental. Nota-se, também, a necessidade de uma solução que envolva as necessidades e expectativas dos usuários, por meio de pesquisas e análises conduzidas junto a eles, como proposto neste trabalho.

Entende-se que este trabalho conseguiu atingir o objetivo proposto ao apresentar como resultado a definição de requisitos fundamentados na revisão bibliográfica e estudo do objeto necessários para uma solução eficaz de design de interfaces. Esperase que os requisitos aqui apresentados, assim como sua fundamentação, possam contribuir como embasamento para futuros trabalhos que pretendam desenvolver artefatos relacionados aos temas abordados.

\section{BIBLIOGRAFIA}

ABERGO - Associação Brasileira de Ergonomia. O que é ergonomia. s.l.: ABERGO, s.d.

BRASIL. Decreto no 24.645 , de 10/JUL/1934.

CFMV. Declaração dos direitos dos animais. s.d. $16^{\circ}$ Ergodesign - Congresso Internacional de Ergonomia e Usabilidade de Interfaces Humano Tecnológica: Produto, Informações Ambientes Construídos e Transporte

$16^{\circ}$ USIHC - Congresso Internacional de Ergonomia e Usabilidade de Interfaces Humano Computador

CINAHPA | 2017 - Congresso Internacional de Ambientes Hipermídia para Aprendizagem.

DAMASCENO, A. Webdesign: Teoria e Prática. Florianópolis: Visual Books, 2003.

\section{GALITZ, W. The Essential Guide to User}

Interface Design: An Introduction to GUI Design Principles and Techniques. 2. ed. New York: John Wiley \& Sons, 2002.

\section{LÉVY, P. As Tecnologias da Inteligência: $O$}

Futuro do Pensamento na Era da Informática. São Paulo: Editora 34, 1998.

MUNARI, B. Das coisas nascem coisas. São Paulo: Martins Fontes, 1998.

NIELSEN, J. Usability 101: Introduction to Usability. On-line: Nielsen Norman Group, 2012.

OSÓRIO, A. Posse responsável: moral, ciência e educação ambiental em um grupo de protetores de gatos de rua. R@U-Revista de Antropologia Urbana, v.3, n.2, p. 51-75, 2011.

PASSOS, R. Design da informação: um modelo para configuração de interface natural, 2014, 231p. Tese (Doutorado em Design) - Departamento de Comunicação e Arte, Universidade de Aveiro, Aveiro - Portugal, 2014.

PRIMO, A. Interação Mútua e Interação Reativa: uma proposta de estudo. Revista da Famecos, n.12, p.81-92. Universidade Federal do Rio Grande do Sul, 2000.

SAMARA, T. Grid: construção e desconstrução. São Paulo: Cosac Naify, 2013.

SIMÃO, J. P. S. et al. Utilização de Experimentação Remota Móvel no Ensino Médio. RENOTE- Revista Novas Tecnologias na Educação, v.11, n.1, p.4-5, UFRGS, 2013.

WINCKLER, M.; PIMENTA, M. S. Avaliação de usabilidade de sites web. Escola de Informática da SBC SUL (ERI 2002). ed. Porto Alegre:

Sociedade Brasileira de Computação (SBC), v. 1, p. 85-137, 2002.
Realização:

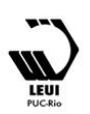

